\title{
Epidemiology and Clinical Characteristics of Patients with Glaucoma in a Hospital Based Population in Central India
}

Dr. Sonalee Mittal*, Dr. Dinesh Mittal

Drishti the Vision Eye Hospital, DF 63, Sceme No 74c, Opp OM Gurudev Complex, Vijay Nagar, Indore, Madhya Pradesh, India

DOI: $10.36347 /$ sjams.2020.v08i07.016

| Received: 30.06 .2020 | Accepted: 08.07.2020 | Published: 18.07.2020

*Corresponding author: Dr. Sonalee Mittal

Purpose: To assess Demographic and Clinical Characteristics of Glaucoma patients in 1345 patients seen at Drishti The Vision Eye Hospital between January 2018 to December 2019. Materials and Methods: Medical charts of patients with Primary Open- Angle Glaucoma (POAG), Primary Angle Closure Glaucoma (PACG), and Secondary Glaucoma (SG) were reviewed. The main outcome measures of patients with Glaucoma included Basic Demographic Data (Age at Presentation, Gender, and Residence), Clinical Characteristics (Vision, Intraocular Pressure, Fundus Photographs and Visual Fields), and Previous History (Diabetes Mellitus, Injury, Hypertension, Cardiovascular Disease, Smoking, and use of Alcohol). Results: Data from 1345 Glaucoma patients were reviewed, of which POAG, PACG and SG patients accounted for 40\% (538 patients) and 44\% (592 patients) and16\% (215 patients) respectively. Female Gender, Age, Cardiovascular Disease, and Hypertension were associated with PACG. POAG was related to Family History, Myopia and Age. There was Positive Correlation between SG and history of Injury and Diabetes Mellitus. In the Secondary Glaucoma Subgroup, 33 subjects had Aphakia,23 had Pseudophakia, 51 Corneo Iridic Scar, 27 had Uveitic Glaucoma, 33 eyes had Post Traumatic Glaucoma and 35 eyes had Neovascular Glaucoma,15 eyes Secondary to uncontrolled Diabetes Mellitus and 17 eyes Secondary to untreated Ischaemic CRVO and 8 eyes had long history of use of Steroids. Conclusion: PACG and POAG are almost prevalent equally in this part of country. We recommend Applanation Tonometry, Gonioscopy, Disc Evaluation, and Perimetry should be incorporated in the Detection Protocol for Glaucoma.

Keywords: Clinical Characteristics, Epidemiology, Glaucoma, Retrospective Study.

Copyright @ 2020: This is an open-access article distributed under the terms of the Creative Commons Attribution license which permits unrestricted use, distribution, and reproduction in any medium for non-commercial use (NonCommercial, or CC-BY-NC) provided the original author and source are credited.

\section{INTRODUCTION}

Epidemiological studies provide us with insights into the amount of disease and its risk factors. Glaucoma is the leading cause of Irreversible Blindness in the world. In 2015, Visual Impairment secondary to Glaucoma accounted for $8.49 \%(2.99 \%-15.66 \%)$ of the world's blindness [1]. The number of people with Glaucoma is expected to increase from 64.3 million in 2013 to 76 million in 2020 and to 111.8 million by $2040 .^{2}$ The majority of adults with Glaucoma live in Asia and Africa [2]. In 2013, the pooled overall Glaucoma prevalence in Asia was 3.54\% [3]. Asia have disproportionately large number of persons with Glaucoma, mostly with Angle-Closure Disease. The Indian report [4] had estimated that 11.2 million people lived with Glaucoma in the year 2009, including 6.48 million people with primary open-angle Glaucoma (POAG) and 2.54 million people with primary angle- closure Glaucoma (PACG). The irreversible and relatively asymptomatic nature of damage caused by Glaucoma makes it a greater public health challenge than Cataracts, which is the leading cause of blindness globally [4]. India was the first country to launch the National Programme for Control of Blindness in 1976 with the goal of reducing the prevalence of blindness. Glaucoma is responsible for $5.5 \%$ prevalence of blindness in the population.

Glaucoma is not a single disease process but a group of disorders characterized by a Progressive Optic Neuropathy resulting in a characteristic appearance of the Optic Disc and a specific pattern of Irreversible Visual Field Defects that are associated frequently but not invariably with raised Intraocular Pressure (IOP). It has now been recognized that Progressive Optic Neuropathy results from the death of Retinal Ganglion Cells (RGCs) in a typical pattern which results in characteristic Optic Disc Appearance and specific Visual Field Defects. 


\section{OphTHALMic Examinations}

Various Ophthalmic Examinations and Diagnostic Procedures were done in the following order:

1. Ocular and Medical History

2. Refraction and Recording of Best-Corrected Visual Acuity

3. Slit Lamp Biomicroscopy, including Van Herick Grading of The Angle of The Anterior Chamber Angle.

4. Applanation Tonometry: IOP recording with the Goldmann Applanation Tonometer.

5. Gonioscopy: A Goldmann 3-Mirror Lens Hand-Held Gonioscope (Volk Optical Inc) was used, and the angle was graded according to the Shaffer System. Gonioscopy was performed in dim ambient illumination with a small slit that did not fall on the pupil. An angle was considered occludable if the pigmented trabecular meshwork was not visible in $>270^{\circ}$ of the angle in dim illumination. All subjects with occludable angles in one or both the eyes were deemed to have primary angle closure disease (PACD). If the angle was occludable, indentation gonioscopy was performed, and the presence or absence of
Peripheral Anterior Synechia was recorded. Laser iridotomy was performed in subjects with occludable angles after obtaining their consent. The rest of the examination was deferred to another convenient date following laser iridotomy

6. Fundus examination. Optic disc evaluation was done using Slitlamp Biomicroscopy with + 78D lens and Fundus Zeiss Camera. The vertical cup- disc ratio (VCDR) was recorded, and a special note was made of Peripapillary Atrophy and Optic Disc/Peripapillary Hemorrhage, Bayoneting Sign, Baring of Circumlinear Vessels, and Laminar Dot Sign.

7. Static Perimetry with Zeiss Perimeter: Visual field evaluation was done with the Zeiss Humphrey Systems perimeter. All subjects underwent the N-30 threshold test. Subjects with unreliable performance were recalled 2 weeks later to repeat the test, and the repeat test results were included in the analysis.

Blood pressure recording were also made.

Diagnostic definition of Glaucoma was based on the ISGEO recommendations [5, 6].

\section{RESULTS}

Table-1: Distribution of Type of Glaucoma

\begin{tabular}{|l|l|l|l|}
\hline \multicolumn{4}{|l|}{ Distribution of Type of Glaucoma (Patients Number) } \\
\hline & MALE & FEMALE & TOTAL \\
\hline POAG & 250 & 288 & 538 \\
\hline PACG & 270 & 322 & 592 \\
\hline SG & 105 & 110 & 215 \\
\hline All Types & 625 & 720 & 1345 \\
\hline
\end{tabular}

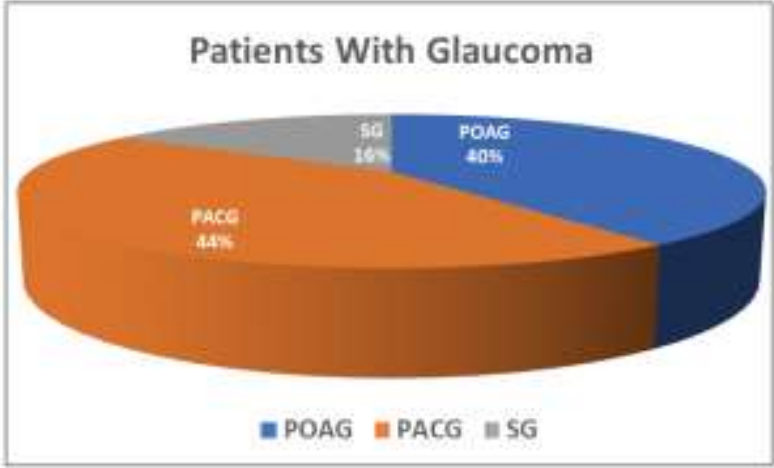

Chart-1: Patients with Glaucoma

Distribution Of Type of Glaucoma

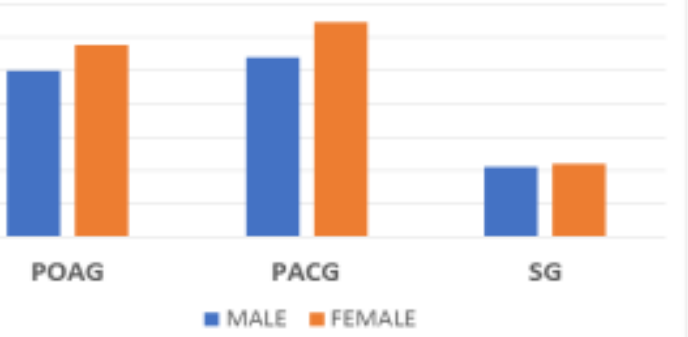

Chart-2: Distribution of Type of Glaucoma 
Table-2: Distribution of vertical cup-disc ratio (VCDR) in subjects with Glaucoma

\begin{tabular}{|l|l|l|l|l|l|l|}
\hline \multicolumn{6}{|c|}{ Distribution of vertical cup-disc ratio (VCDR) in subjects with Glaucoma } \\
\hline & 0.6 & 0.7 & 0.8 & 0.9 & 1 & \\
\hline POAG & $\mathbf{8 0}$ & 100 & 110 & 110 & 138 & 538 \\
\hline PACG & 188 & 162 & 92 & 88 & 62 & 592 \\
\hline SG & 35 & 42 & 43 & 48 & 47 & 215 \\
\hline
\end{tabular}

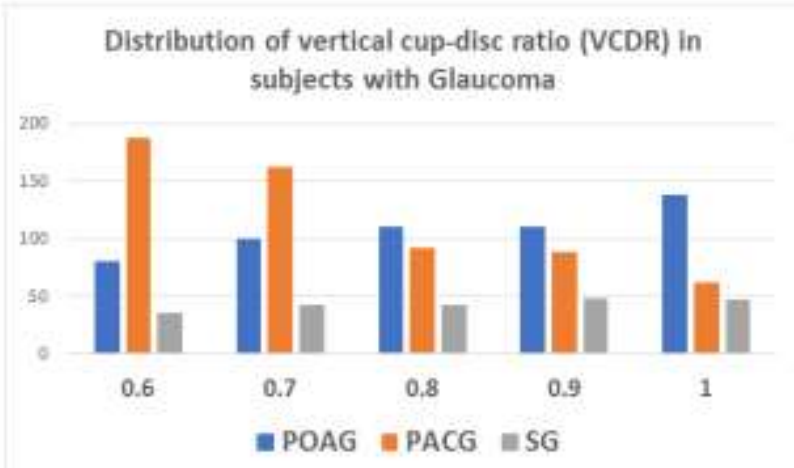

Chart-3: Distribution of vertical cup-disc ratio (VCDR) in subjects with Glaucoma

Table-3

\begin{tabular}{|c|c|c|c|c|c|c|}
\hline \multicolumn{7}{|c|}{ Age distribution of subjects detected with Glaucoma } \\
\hline & 40-50 Age & Age & Age & $>70$ Yrs & Total & \\
\hline POAG & 80 & 96 & 152 & 210 & 538 & \\
\hline PACG & 212 & 168 & 122 & 90 & 592 & \\
\hline SG & 35 & 57 & 55 & 68 & 215 & \\
\hline
\end{tabular}

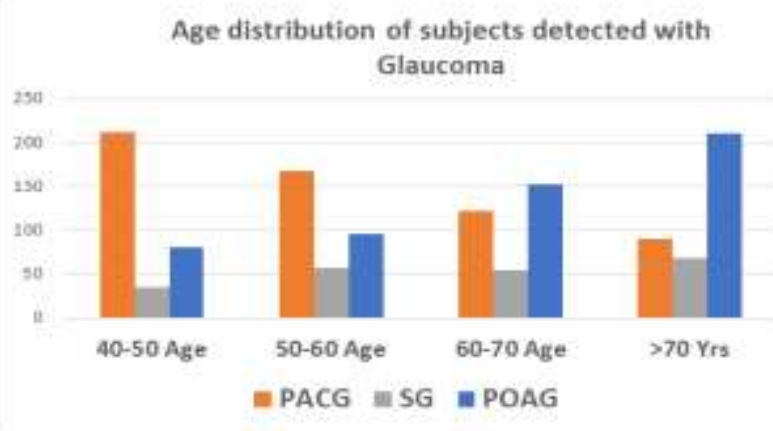

Chart-4: Age Distribution of Subjects Detected with Glaucoma

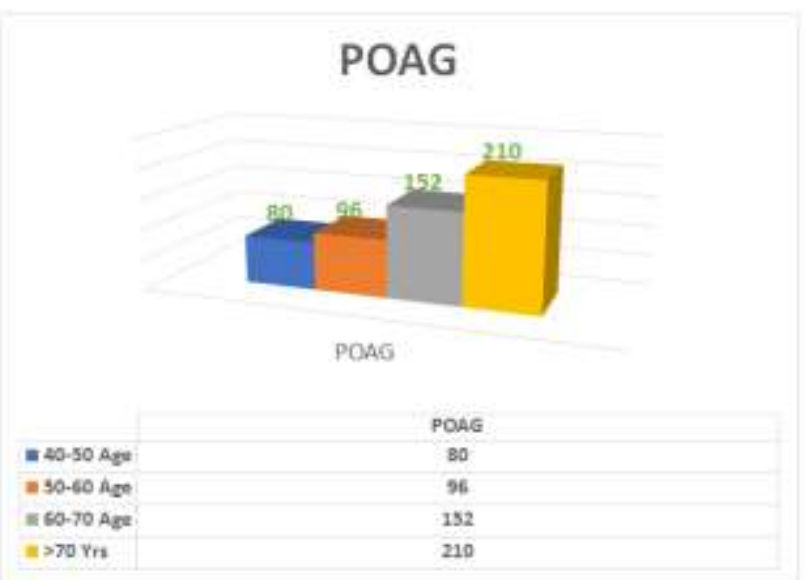

Chart-5: Age Distribution of Subjects Detected with POAG 
Table-4: The distribution of intraocular pressure (IOP) in subjects WITH Glaucoma

\begin{tabular}{|l|l|l|l|l|}
\hline \multicolumn{5}{|c|}{ The distribution of intraocular pressure (IOP) in subjects WITH Glaucoma } \\
\hline & $23-26$ IOP & $27-30$ IOP & $31-34$ IOP & $>35$ \\
\hline POAG & 123 & 173 & 153 & 89 \\
\hline PACG & 73 & 77 & 183 & 259 \\
\hline SG & 35 & 57 & 53 & 70 \\
\hline
\end{tabular}

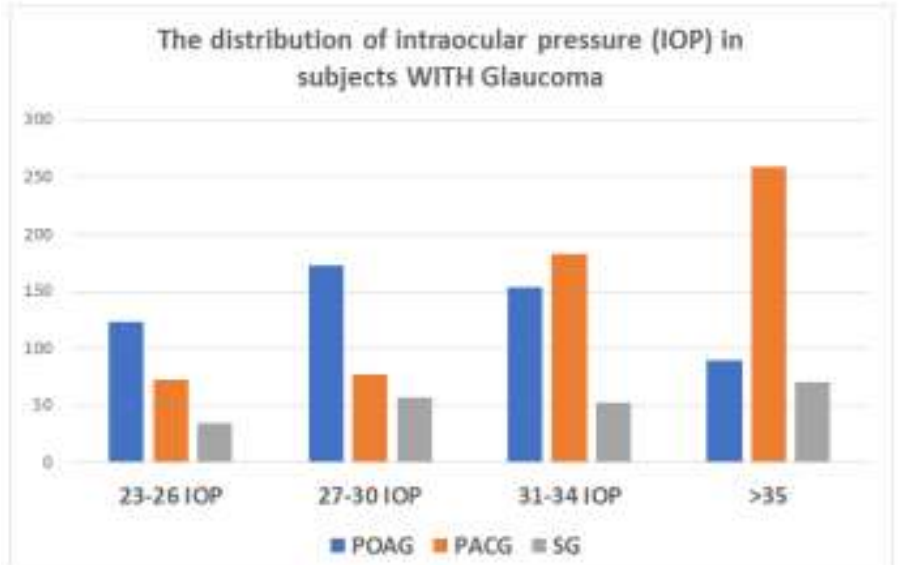

Chart-6: The distribution of intraocular pressure (IOP) in subjects WITH Glaucoma

Table-5: Etiology of Different Secondary Glaucoma

\begin{tabular}{|l|l|}
\hline \multicolumn{2}{|l|}{ Etiology Of Different Secondary Glaucoma } \\
\hline & NUMBER \\
\hline Post-traumatic & 33 \\
\hline Corneo-iridic scar & 51 \\
\hline Aphakic & 33 \\
\hline Pseudophakic & 23 \\
\hline Neovascular & 35 \\
\hline Uveitic & 27 \\
\hline Steroid Induced Glaucoma & $\mathbf{8}$ \\
\hline Miscellaneous & 5 \\
\hline & 215 \\
\hline
\end{tabular}

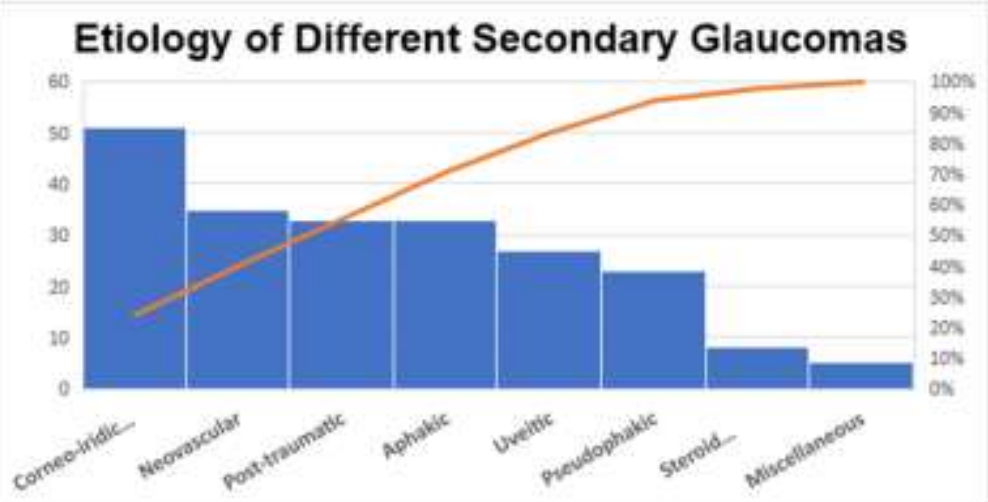

Chart-7: Etiology of Different Secondary Glaucoma

Table-6: Prevalence of POAG in India

\begin{tabular}{|l|l|l|l|}
\hline \multicolumn{4}{|c|}{ Prevalence of POAG in India [7-9] } \\
\hline & APEDS \% & CGS $($ Rural) \% & CGS (Urban) \% \\
\hline 40-49 years & 1.27 & 0.63 & 2.26 \\
\hline 50-59 years & 2.31 & 1.62 & 3.57 \\
\hline 60-69 years & 4.89 & 2.58 & 4.08 \\
\hline$>70$ years & 6.32 & 3.25 & 6.42 \\
\hline
\end{tabular}


Table-7: Prevalence of Angle Closure Disease in India

\begin{tabular}{|l|l|l|l|l|l|l|l|l|l|}
\hline \multicolumn{9}{|c|}{ Prevalence of Angle Closure Disease in India [7-9] } \\
\hline & \multicolumn{1}{|l|}{ APEDS } & \multicolumn{1}{l|}{ CGS (Rural) } & \multicolumn{3}{l|}{ CGS (Urban) } \\
\hline Age years & PACG & PAC & PACG & PAC & PACS & PACG & PAC & PACS \\
\hline 40-49 years & 0.00 & 0.76 & 0.44 & 0.38 & 4.29 & 0.07 & 1.27 & 4.86 \\
\hline 50-59 years & 1.54 & 3.08 & 1.02 & 0.81 & 7.31 & 0.63 & 3.30 & 7.77 \\
\hline 60-69 years & 2.17 & 3.80 & 1.01 & 1.01 & 7.96 & 2.21 & 4.19 & 9.27 \\
\hline$>70$ years & 3.16 & 5.26 & 1.73 & 1.08 & 7.58 & 1.48 & 3.21 & 9.38 \\
\hline
\end{tabular}

Several studies have shown that the prevalence of Glaucoma [7-9] increases with Age. Our study also showed a similar trend with the prevalence being more than $4 \%$ in those aged $\geq 70$ years. CGS (The Chennai Glaucoma study) also found similar results with subjects over 70 years being five times more likely to have POAG than those younger than 50 years.

We divided all the patients into three subgroups such as Primary Open-Angle Glaucoma (POAG), PACG, Secondary Glaucoma (SG), However, PACG is more common in this region than previously thought, and the current study strongly recommends that gonioscopy should be made an integral part of the routine ophthalmological evaluation. IOP is universally recognized as one of the most important modifiable risk factors for the development of POAG, our study also concludes similarly. The average IOP across various age groups was statistically significantly higher $(P<$ 0.001 ) in those with POAG as compared to normal subjects.

Discussion The National Programme for Control of Blindness, though started in 1976 has not been developed as Comprehensive Eye Care Program and this has led to a delay in the detection and management of patients with Glaucoma. Widely-tipped as Silent Thief of Sight owing to its largely asymptomatic nature, population-based studies have revealed that more than 90 percent of Glaucoma in the developing world remains undetected. Residency and postgraduate training programs need to be well equipped to train graduating ophthalmologists to increasingly focus on case detection of Glaucoma by the Opportunistic Screening, which remains the Single Most Effective Means of early detection of Glaucoma for instituting an appropriate management.

Studies have shown that Increasing Age, people residing in Urban Areas, people with Diabetes, those with High Myopia, and people with a Positive Family History of Glaucoma have Higher Risk for POAG [10-12]. Similarly, Risk Factors for PACG include Advanced Age, Female Gender, those with Family History of Glaucoma, Short Stature, People with Narrow Palpebral Aperture, and people with High Hyperopia. The target coverage must be more intensive with increasing age since the prevalence of POAG shows a significant rise with age. Studies have shown a POAG prevalence of $3.45 \%$ for those over 40 years,
$5.11 \%$ for those over 50 years, and $7.50 \%$ for those over 60 years.

Secondary Glaucoma was present in $16 \%$ (215 patients) of all the patients. In the Secondary Glaucoma Subgroup, 33 subjects had Aphakia, 23 had Pseudophakia, 51 Corneo Iridic Scar, 27 had Uveitic Glaucoma, 33 eyes had Post Traumatic Glaucoma and 35 eyes had Neovascular Glaucoma, 15 eyes Secondary to uncontrolled Diabetes Mellitus and 17 eyes Secondary to untreated Ischaemic CRVO. Steroidinduced Glaucoma was present in 8 cases. Of all cases of steroid-induced cases, $50 \%$ were avoidable and were due to the use of treatment for ocular allergies or postoperatively or drops used over the counter without prescription. The rest were due to treatment for systemic conditions such as sarcoidosis, scleroderma, primary ulcerative colitis, nephritic syndrome and uveitis. These could also have been avoidable if the concerned physicians treating them for these systemic diseases were educated on the possibility of steroidinduced IOP elevation and Glaucoma and had either warned the patients or had referred them to ophthalmologists for interventions.

Uveitic Glaucoma followed attacks of Anterior Uveitis in $90 \%$ cases, and the rest were seen in Panuveitis. Bilateral involvement was seen in $50 \%$ of cases. Pseudophakic Glaucoma affected the older population, $80 \%$ of patients being above 40 years of age. Most cases were unilateral (93\%). A large number of eyes $(37 \%)$ had either Anterior Chamber Intraocular Lens (IOL) or a Posterior Chamber IOL placed in the Anterior Chamber or a Pupillary Capture of IOL. Ninety per cent cases had undergone a Complicated Cataract Surgery. Glaucoma secondary to Corneal Pathologies was most frequent. Fifty per cent of these cases were less than 40 years of age. The most common etiology was a healed corneal ulcer $(50 \%) ; 10 \%$ were Post Traumatic. All eyes had a vision of $<20 / 200$, and fundus evaluation was not possible due to the presence of the opacity.

Unlike most of the other disease states, Glaucoma is not one disease entity, but a composite mixture of different pathologies: POAG, ACG, SG, as well as Congenital Glaucoma. Thus, establishing a uniform case definition is not possible. Though, increased IOP is a major risk factor for development of Optic Neuropathy it is seen, that Glaucomatous damage occurs even at lower IOP values. On the other hand, 
increased IOP may not cause any damage in certain individuals. So, taking a single IOP measurement may not be of very helpful in actually labeling an individual to be having Glaucoma. Next Ophthalmoscopy Examination can also give rise to high percentage of false positive and negative results. Mass Visual Field testing has been shown to be a potentially accurate and efficient means for screening by some authorities, but 40 percent nerve fibers may already be lost by the time, a Functional Field Defect is detected. Here addition of OCT for diagnosis of Glaucoma at Pre Perimetric-Stage is a welcome addition. Moreover, to screen for Angle Closure Glaucoma, Gonioscopy facilities should also be available.
The Simplistic Goal of Community Eye Care in Glaucoma is to diagnose and treat. Mass community screening is unlikely to be cost effective, and in addition, there is a lack of trained personnel and required infrastructure. An Opportunistic Screening is a good alternative. This could be eased with a Comprehensive Eye Examination of all people above the age group of 40 reporting to the clinic. This includes measurement of Presenting and Best Corrected Visual Acuity, Slit- Lamp Examination (including Van Herick test and IOP measurement, Gonioscopy (if required), and Dilated Stereoscopic Evaluation of the Optic Disc and Retina.

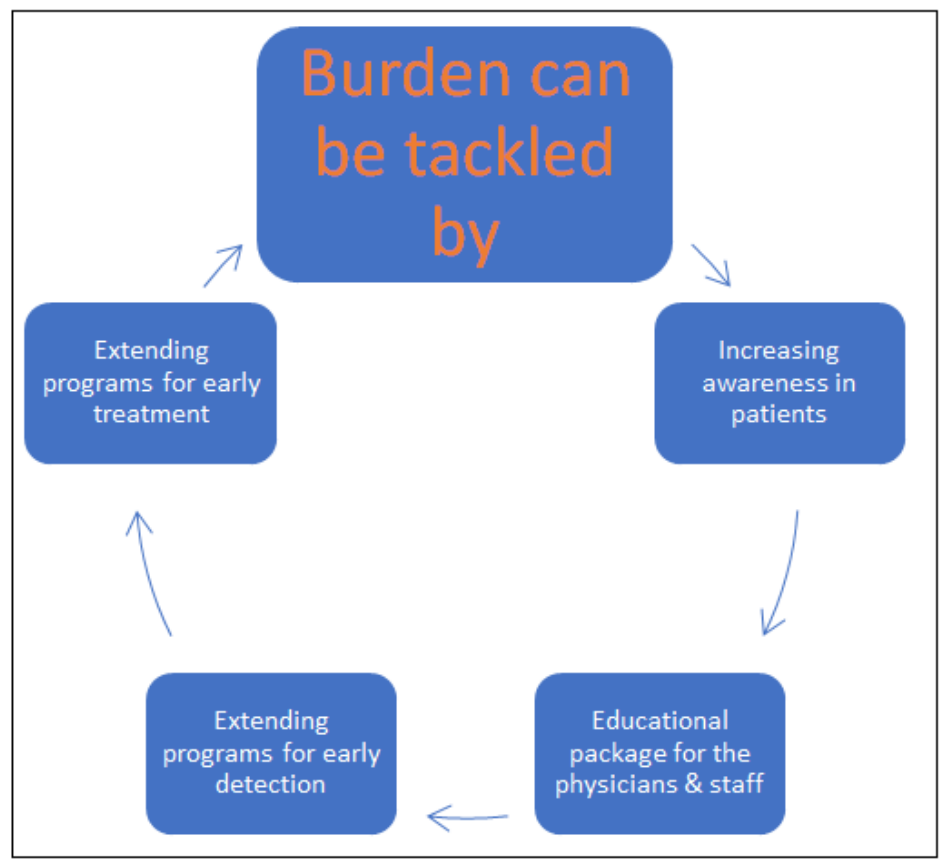

We strongly recommend Applanation Tonometry, Gonioscopy, Disc Evaluation, and Perimetry should be incorporated in the detection protocol for Glaucoma. Let us all Ophthalmologists join hands together for Preventing, Screening, Diagnosing and Treating Glaucoma early to make our society free of Preventable Irreversible Glaucoma Induced Blindness.

\section{REFERENCES}

1. Flaxman SR, Bourne RR, Resnikoff S, Ackland P, Braithwaite T, Cicinelli MV, Das A, Jonas JB, Keeffe J, Kempen JH, Leasher J. Global causes of blindness and distance vision impairment 19902020: a systematic review and meta-analysis. The Lancet Global Health. 2017 Dec 1;5(12):e1221-34.

2. Tham YC, Li X, Quigley HA, Aung T, Cheng CY. Global prevalence of Glaucoma burden through 2040: A systematic review and meta- analysis. Ophthlmology. 2014; 121:2081-90.

3. Chan EW, Li X, Tham YC, Liao J, Wong TY, Aung T, Cheng CY. Glaucoma in Asia: regional prevalence variations and future projections. British Journal of Ophthalmology. 2016 Jan 1;100(1):78-85.

4. George R, Ve RS, Vijaya L. Glaucoma in India: Estimated burden of disease. J Glaucoma. 2010; 19:391- 7.

5. Paul C, Sengupta S, Choudhury S, Banerjee S, Sleath BL. Prevalence of Glaucoma in Eastern India: The Hooghly river Glaucoma study. Indian J Ophthalmol. 2016; 64:578-83.

6. Foster PJ, Buhrmann R, Quigley HA, Johnson GJ. The definition and classification of Glaucoma in prevalence surveys. Br J Ophthalmol. 2002; $86: 238-42$.

7. Vijaya L, George R, Baskaran M, Arvind H, Raju P, Ramesh SV, Kumaramanickavel G, McCarty C. Prevalence of primary open-angle glaucoma in an urban south Indian population and comparison with a rural population: the Chennai Glaucoma Study. Ophthalmology. 2008 Apr 1;115(4):648-54.

8. Vijaya L, Rashima A, Panday M, Choudhari NS, Ramesh SV, Lokapavani V, Boddupalli SD, Sunil 
GT, George R. Predictors for incidence of primary open-angle glaucoma in a South Indian population: the Chennai eye disease incidence study. Ophthalmology. 2014 Jul 1;121(7):1370-6.

9. Dandona L, Dandona R, Srinivas M, Mandal P, John RK, McCarty CA, Rao GN. Open-angle glaucoma in an urban population in southern India: the Andhra Pradesh eye disease study. Ophthalmology. 2000 Sep 1;107(9):1702-9.

10. Jiang X, Varma R, Wu S, Torres M, Azen SP, Francis BA, Chopra V, Nguyen BB, Los Angeles Latino Eye Study Group. Baseline risk factors that predict the development of open-angle glaucoma in a population: the Los Angeles Latino Eye Study. Ophthalmology. 2012 Nov 1;119(11):224553.

11. Le A, Mukesh BN, McCarty CA, Taylor HR. Risk factors associated with the incidence of open- angle Glaucoma: The visual impairment project. Invest Ophthalmol Vis Sci. 2003; 44:3783- 9 .

12. Leske MC, Wu SY, Hennis A, Honkanen R, Nemesure B; BESs Study Group. Risk factors for incident open- angle Glaucoma: The Barbados eye studies. Ophthalmology. 2008; 115:85-93. 